\section{The Pion as a Probe of Conventional Nuclear Physics}

\author{
Colin Wilkin, London \\ (Dept. of Physics and Astronomy, University College)
}

periments is typically at least a factor of ten poorer than in the above. This is because the $\pi^{\circ}$ is neutral and both photons from its decay must be measured accurately. Such reactions are, nevertheless, suitable for the hunting of giant resonances which are several $\mathrm{MeV}$ wide. Giant resonances are fundamental to our understanding of nuclear collective motion - they reflect bulk properties of the nucleus rather than the idiosyncrasies of a particular nucleon. Monopole or breathing resonances involve the periodic compression and expansion of the neutron and proton densities in the nucleus, but the isovector type, where the neutrons and protons oscillate out of phase, have proved very difficult to identify in the past. The probe used to excite them must be capable of transferring isopin and preferably should be strongly interacting and have spin zero in order to suppress the small angle production of the giant dipole resonances which are well known from photo-absorption. The background can be further diminished if charge exchange is also required and this suggests that the states be looked at via the $\left(\pi^{-}, \pi^{\circ}\right)$ reaction. [The alternative $\left(\mathrm{K}^{-}, \mathrm{K}^{\circ}\right)$ reaction could give a better energy resolution but such studies must await the building of a kaon factory!]

On the basis of a simple hydrodynamical model, the resonance energy is estimated to be of the order of 170 $\mathrm{MeV} / \mathrm{A}^{1 / 3}$ although this value is modified a little in more microscopic calculations. It was therefore gratifying when recent experiments $^{2}$ ) at LAMPF with the $\pi^{0}$ spectrometer clearly identified such states, starting from the targets ${ }^{120} \mathrm{Sn}$ and ${ }^{90} \mathrm{Zr}$, close to the predicted positions. These findings depend little upon the pion-nucleus scattering theory. In the $100-200 \mathrm{MeV}$ range of energies the middle of the nucleus is black to pions due to the production of the delta resonance (see p. 2) and the multipolarity of the state can be seen by inspection of the angular distribution using the Blair strong absorption model. More detailed calculations do however reproduce the magnitude of the observed crosssections.

It is expected that these and similar measurements will help resolve uncertainties associated with isospin mixing

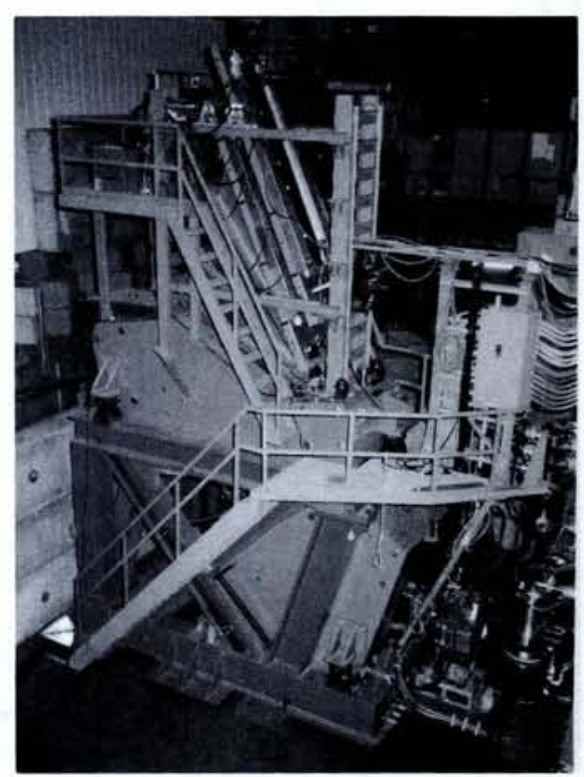

The SIN Universal Spectrometer Installation (SUSI) mounted on a pion beam-line of the SIN isochronous synchrocyclotron which accelerates protons up to an energy of 590 MeV. The pion intensity at the experimental target is $10^{6}-10^{7} \mathrm{pion} / \mathrm{s}$ and the overall resolution of the spectrometer is about $1.5 \times 10^{-3} \mathrm{\partial p} / \mathrm{p}$.

and provide constraints to the isovector components of the effective nucleonnucleon interaction in the nucleus.

\section{Nuclear Radii}

One of the major features of the pion as a probe is the strong dependency of its interactions on energy: in contrast to the delta region, where the nucleus looks like a black disk, at low energies the mean free path of the pion is quite long. The pion-nucleus interaction has also an important isospin dependence which suggests that a comparaison of the elastic scattering of $\pi^{+}$and $\pi^{-}$by a nucleus should give information on the relative proton and neutron densities in its interior. Scattering theory is not yet sufficiently advanced to yield precise absolute nuclear parameters from such data, but having adjusted the strength of the effective interaction on an isoscalar nucleus which has equal numbers of neutrons and protons, for example ${ }^{12} \mathrm{C}$, it is possible to derive the relative density differences of ${ }^{13} \mathrm{C}$ experimentally. This approach has been used extensively with other nuclei at TRIUMF in Vancouver over the energy range $30-50$ MeV. By extracting the various functional forms for the optical potential from the current gamut of theoretical models, realistic error bars which include some uncertainty from the scattering theory can be constructed.

Relatively simple experiments ${ }^{3}$ ) seem to give the differences between the
The energy resolution achieved in single charge exchange $\left(\pi^{ \pm}, \pi^{\circ}\right]$ ex- 
r.m.s. radii of the neutrons in the $\left({ }^{13} \mathrm{C},{ }^{12} \mathrm{C}\right)$ and $\left({ }^{18} \mathrm{O},{ }^{16} \mathrm{O}\right)$ pairs as $0.04 \pm 0.03 \mathrm{fm}$ and $0.21 \pm 0.03 \mathrm{fm}$ respectively. The numbers are close to theoretical estimates and further confidence in the technique comes from a comparaison of the charge radii of ${ }^{12} \mathrm{C}$ and ${ }^{1} \mathrm{~B}$ which agrees with that deduced with electromagnetic pro bes.

More advanded low energy pion spectrometers are being commissioned at all three meson factories and so we can expect more refined experiments in this domain soon.

\section{Charge Symmetry of Nuclear Forces}

In the analyses of the experiments described so far, it has been assumed that the interactions conserve isospin, but the pion is an ideal probe to test for isospin violation in nuclear forces. Since the deuteron is symmetric with regard to the interchange of its constituent proton and neutron, from the charge symmetry of nuclear forces it would be expected that, apart from Coulomb effects, its scattering of the charge conjugates $\pi^{+}$ and $\pi^{-}$would be identical.

When seeking to verify whether this is found in practice, experimental chargedependent biasses can best be reduced by performing total cross-section measurements Results of such measure ments at $\mathrm{SIN}^{4}$ ) covering the energy range $70-350 \mathrm{MeV}$ where the production of $\triangle$ resonances dominates the interaction, indicated that there are indeed $\pi^{+}, \pi^{-}$differences amounting to a few per cent. Unlike the case of the protonproton versus neutron-neutron scattering lengths which is the classic test of charge symmetry, Coulomb effects are, in the pion interaction, relatively small. Consequently, there is less uncertainty introduced when correcting for them. The strongly energy-dependent signal that remains, could best be understood as a mass splitting in the multiplet of the pion-nucleon delta resonances with the $\Delta^{++}$being lighter than the $\Delta^{-}$by about $4 \mathrm{MeV} / \mathrm{c}^{2}$ and with a corresponding width change. Very similar results have since been obtained from elastic scattering ${ }^{5}$ ) though the Coulomb corrections are there somewhat larger. This clear charge symmetry violation is in accord with the naive quark model estimate of three times the neutron-proton mass difference, and with more modern particle physics estimates.

A large part of the nucleon-nucleon force arises from virtual pion-nucleon scattering where a pion is shaken off from one nucleon, scatters from the second and is reabsorbed by the first. However it seems that little of the charge symmetry violation in pion-nucleon scattering propagates through the nucleon-nucleon system just because the difference in the delta masses compensates that of the nucleons.

\section{Isospin Mixing of Nuclear Levels}

Charge symmetry violation in pion scattering by nuclei more complex than the deuteron can also occur through isospin impurities in the nuclear states. In the absence of any symmetry breaking, the excitation of any level of a selfconjugate nucleus such as ${ }^{16} \mathrm{O}$ should be the same in $\pi^{+}$and $\pi^{-}$scattering. This nucleus possesses three spin- 4 negative parity levels in the $18-20 \mathrm{MeV}$ range, two with isospin zero and one with isospin one. In an experiment carried out with $164 \mathrm{MeV}$ pions at LAMPF, it was found that the lower isoscalar level was excited $60 \%$ more strongly by $\pi^{+}$and the upper similarly by $\pi^{-}$, whereas the isovector level was equally populated in $\pi^{+}$and $\pi^{-}$scattering. The authors could explain these ratios quantitatively by $a \pm 12 \%$ contamination of the isoscalar levels by the isovector, and the

\section{New Nuclear Physics Titles from Reidel}

\section{Handbook of Materials Testing Reactors}

and Associated Hot Laboratories in the European Community

Nuclear Science and Technology

Edited by PETER VON DER HARDT and HEINZ RÖTTGER

1981, viii $+152 \mathrm{pp}$.

Cloth DfI. 55,- / US \$ 24.00

ISBN 90-277-1347-2

This useful handbook documents the facilities of EEC-country testing nuclear reactors, and shows that they are capable of handling any type of work that can be done with neutrons: fundamental and tech. nological materials research; radioisotope production for industrial, medical and scientific purposes; nuclear and non-nuclear application of neutron radiography; solid state and nuclear physics research; activation analysis; and training and education.

\section{Neutron}

\section{Radiography}

Proceedings of the First World Conference, San Diego, California, U.S.A., December 7-10, 1981

Edited by JOHN P. BARTON and PETER VON DER HARDT

1983, xvii $+1073 \mathrm{pp}$

Cloth DfI. 290,- / US \$ 126.00

ISBN 90-277-1528-9

A large proportion of the papers presented here concern applications

\section{Reidel Publishing Company}

P.O. Box 17, 3300 A A Dordrecht, Holland

190 Old Derby Street, Hingham, MA 02043, U.S.A. for inspection of nuclear fuel, where high precision dimensional measurements and track etch imaging are receiving growing atten tion.

Nuclear Data for Science and Technology

Proceedings of the International Conference, Antwerp, 6-10 September, 1982

Edited by K. H. BÖCKHOFF

1983, xviii $+1056 \mathrm{pp}$.

Cloth DfI. 275,- / US \$ 120.00

ISBN 90--277-1560-2

Papers deal with the design, safety and fuel cycle of fission reactors, the conceptual design of fusion reactors, nuclear data in biomedicine, astrophysics and solid-state research.

\section{Irradiation Technology}

Proceedings of an International Topical Meeting, Grenoble, France, September 28-30, 1982

Edited by PETER VON DER HARDT and HEINZ ROTTGER 1983, xiv +759 pp

Cloth Dfl. 230,- / US \$ 100.00 ISBN 90-277-1568-8

These papers demonstrate a high level of technological devclopment as opposed to a decreasing number of large materials testing reactors available. 
parameters of their three level model have since been checked in proton scattering and most dramatically electron scattering ${ }^{7}$ ) experiments. In the latter, the lower state is enhanced by a factor of five by the mixing, whereas the upper state is suppressed by a factor of ten and has so for not even been seen. The technique for measuring isospin mixing has also been tested on other nuclei such as ${ }^{28} \mathrm{Si}$ with similar success.

The nuclear information gleaned from the examples outlined here depends relatively little upon the ambiguities of reaction theory since distortion effects should be very similar for the different charge states of the pion. There is a little more model dependence in other phenomena such as the extraction of information on the convection current from inelastic transitions induced by the strong energy dependence of the effective interaction. There is a wealth of other information associated with more exotic reactions such as pion absorption, but this may be dominated by more exotic nuclear physics and we are still at the stage of exploring the reaction mechanism. Pions are expensive but they have many features which make them rather special nuclear probes.

\section{REFERENCES}

1. Nann H., Proc. VI Int. Conf. on Atomic Masses, East Lansing, 1979: Seth K., 4th Int. Conf. on Nuclei far from Stability, CERN $81-09$ (1981) 685

2. Bowman J.D. et al., Phys. Rev. Letters 50 (1983) 1195; but see also: Bowan J.D. Johnson M.B. and Negele J.W., Phys. Rev. Lett.46 (1981) 1614.

3. Johnson R.R. et al., Phys. Rev. Lett. 43 (1979) 844.

4. Pedroni E. et al., Nucl. Phys. A300 (1978) 321

5. Masterson T.G. et al., Phys. Rev. C26 (1982) 2091.

6. Holtkamp D.B. et al., Phys. Rev. Lett. 45 (1980) 420.

7. The experimental situation is well summarised by: Petrovich F. and Love W.G. Nucl. Phys. A354 (1981) 499c.

\section{Call for Nominations}

A new Board of the Nuclear Physics Division is elected every four years and the next election is due this year. Ten members are elected and up to a further seven may be co-opted to obtain geographical and subject balance of representation.

Nominations for the new Board should be sent to the present Secretary:

Dr. J.F. Sharpey-Schafer,

Physics Department, The University,

POB 147, Liverpool L69 3BX, England.

\section{Closing date for nominations} 1 March, 1984

Nominations should include the signed consent of the nominee and the signatures of FOUR Ordinary Individual Members of the EPS.

Divisional members nominated by the present Board are:
A. Berinde (Bucharest)
P. Glaudemans (Utrecht)
G. Goldring (Tel Aviv)
I. lori (Milano)
J. Kantle (Jyväskylä)
I. Sick (Basel)
G. Tibell (Uppsala)
I. Ulehla (Prague)
H. Zingl (Graz)

\section{Formal Announcement}

The triennial General Meeting of the members of EPS will be held in Prague on Tuesday, 28 August 1984, beginning at 9 h. 50 and will be followed by the Hewlett-Packard Euro- physics Prize award ceremony and lecture.

Applicants for membership awaiting acceptance will be admitted provided that the first membership fee has been paid.

Members are invited to make proposals for topics to be placed on the Agenda in time for publication in the May issue of Europhysics News.

\section{Preliminary Agenda}

1. Report of the President

2. Report of the Secretary

3. Report of the Treasurer

4. Discussion of subjects of general interest according to suggestions and proposals put forward by members.

\section{Hewlett-Packard Prize}

The 1984 Hewlett-Packard Europhysics Prize has been awarded to:

G. Binnig and $H$. Rohrer

both of IBM Research Laboratory, Rüschlikon, for their scanning tunnelling microscope. Details will be given in the February issue.

\section{FACULTES UNIVERSITAIRES NOTRE-DAME DE LA PAIX - NAMUR}

Applications are invited for a PROFESSORSHIP in general physics (undergraduate level) and molecular and laser spectroscopy (graduate and doctorate levels) at the Department of Physics. Duties include also the responsibility for the leadership of an experimental laboratory in high resolution molecular spectroscopy with some developments in laser physics.

The Department of Physics has presently a scientific staff of 30 . Research groups are working in several fields of applied physics (lasers, solids, surfaces and interfaces, development of non-destructive methods of physical analysis using photons, electrons and nuclear projectiles, mathematical physics and theoretical physics of solids), and interdisciplinary work with mathematicians, chemists and biologists.

Applicants must be able to teach in fluent French, and give a general physics course to a large group of undergraduate students.

Enrolment is foreseen as 1 September 1984.

Application, with curriculum vitae, should be sent by 31 May 1984 to:

R.P. R. TROISFONTAINES, Recteur des Facultés Universitaires N.D. de la Paix 61, rue de Bruxelles, B - 5000 - Namur (BELGIUM)

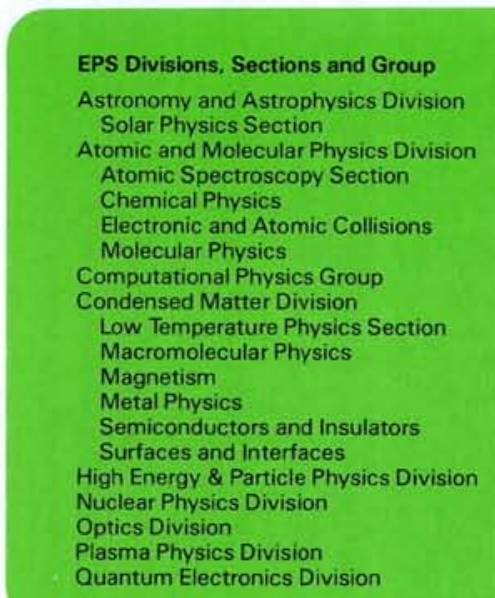

Europhysics News is the official journal of the European Physical Society which comprises 29 National Socie ties, Academies and Group, over 3000 Individual Memties, Academies and Group, over 3000 Individual MemEPS are the General Meeting, Council and an elected Executive Committee responsible for detailed policy. EPS promotes the collaboration of physicists throughout Europe, organising and harmonising conferences and publications, improving physics education, encou raging physics applications, awarding scholarships to sponsored schools in Erice. EPS publishes in addition to EN, Europhysics Conference Abstracts, E. Ed. News and, in collaboration with The Institute of Physics (UK), and, in collaboration with The Institute of Physics (UK), receive EN free of charge (price to institutions: Sw.Fr. 90.-la), rebates on the price of many publications and on conference fees. Annual EPS membership fee for Individual Members belonging to an EPS member society is: Sw.Fr. 40.-; independent members: Sw.Fr 120.: Am. Phys. Soc. members: Sw.Fr. 50.- (\$25).

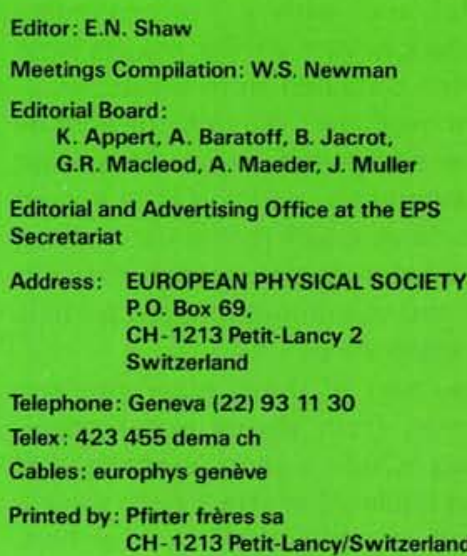

